\title{
Efficient sympathetic motional-ground-state cooling of a molecular ion
}

\author{
Yong Wan, ${ }^{1}$ Florian Gebert, ${ }^{1}$ Fabian Wolf, ${ }^{1}$ and Piet O. Schmidt ${ }^{1,2, *}$ \\ ${ }^{1}$ QUEST Institut, Physikalisch-Technische Bundesanstalt, 38116 Braunschweig, Germany \\ ${ }^{2}$ Institut für Quantenoptik, Leibniz Universität Hannover, 30167 Hannover, Germany
}

(Received 29 January 2015; published 30 April 2015)

\begin{abstract}
Cold molecular ions are promising candidates in various fields ranging from precision spectroscopy and test of fundamental physics to ultracold chemistry. Control of internal and external degrees of freedom is a prerequisite for many of these applications. Motional-ground-state cooling represents the starting point for quantum logic-assisted internal state preparation, detection, and spectroscopy protocols. Robust and fast cooling is crucial to maximize the fraction of time available for the actual experiment. We optimize the cooling rate of ground-state cooling schemes for single ${ }^{25} \mathrm{Mg}^{+}$ions and sympathetic ground-state cooling of ${ }^{24} \mathrm{MgH}^{+}$. In particular, we show that robust cooling is achieved by combining pulsed Raman sideband cooling with continuous quench cooling. Furthermore, we experimentally demonstrate an efficient strategy for ground-state cooling outside the Lamb-Dicke regime.
\end{abstract}

DOI: 10.1103/PhysRevA.91.043425 PACS number(s): 37.10.Rs, 37.10.Mn, 37.10.Pq, 37.10.Ty

\section{INTRODUCTION}

Coherent manipulation of quantum systems has been a goal for a wide range of fields in physics and chemistry. The ability to control internal and external degrees of freedom of atoms has enabled applications such as quantum computation [1-4], quantum simulation [5,6], and quantum metrology [7-12]. The control over the internal degrees of freedom of the atomic species is often accomplished through coherent manipulation using laser, microwave, or radio-frequency (rf) pulses. The external degrees of freedom are typically controlled with various laser cooling techniques. Extending these coherent manipulation techniques to molecular ions promises novel applications including multiqubit quantum memories, quantum information processing $[13,14]$, and ultracold chemistry [15-17]. Moreover, molecular ions are particularly promising candidates for several spectroscopic tests of fundamental physics, ranging from a possible variation of the electronto-proton mass ratio [18-22] over an enhanced sensitivity to a possible dipole moment of the electron [23-27] to parity violation observable through a small energy difference in enantiomers of polyatomic molecules [28-31].

However, controlling the quantum state of molecular ions represents a challenge arising from their rich internal level structure, including rotation and vibration. As a result of the absence of selection rules for vibrational transitions (except for a few special cases with near-diagonal Franck-Condon matrices [32-34]) no closed cycling transitions are available to implement laser cooling. This limitation can be overcome by sympathetic cooling with an atomic species trapped together with the molecule. While sympathetic Doppler cooling of molecular ions has been demonstrated by several groups in the past [35-39], only recently sympathetic ground-state cooling of $\mathrm{CaH}^{+}$using $\mathrm{Ca}^{+}$has been achieved [40]. The approach is identical to previous successful implementations using atomic ions trapped in linear radio-frequency Paul traps [7,41-45].

Besides the lowest achievable mean population of motional states $\bar{n}$, which can reach $\bar{n} \sim 0.001$ for a single ion [46], the time spent on cooling is an important aspect. Short

*piet.schmidt@quantummetrology.de cooling times reduce overhead in the experimental cycle and are relevant for quantum algorithms [45,47], as well as spectroscopy experiments requiring ground-state cooling, such as quantum logic spectroscopy [7,9] and photon recoil spectroscopy [11].

Cooling to the motional ground state of a single mode can be achieved via resolved sideband cooling (SBC), which requires the motional sidebands in the excitation spectrum of the system to be spectrally resolved. It has been demonstrated in various systems ranging from single trapped ions in Paul traps [48-51] to neutral atoms in optical lattices [52,53] or optical tweezers [54] and micromechanical oscillators [55-57]. In the following we will focus on ground-state cooling of trapped and localized one- and two-ion crystals, represented by two-level systems with two metastable electronic states $(|\uparrow\rangle,|\downarrow\rangle)$. The crystals are confined in a harmonic trap with motional Fock states $|n\rangle$, separated by the trap frequency $\omega_{\mathrm{T}}$, as shown in Fig. 1(a). Kinetic energy is removed from the system by selectively driving red-sideband (RSB) transitions involving a change of the electronic state while reducing the motional quantum number, followed by spontaneous emission back to the electronic ground state. In the Lamb-Dicke regime, changes in the motional state upon spontaneous emission are strongly suppressed [58-60].

Sideband cooling is typically implemented in two flavors. In continuous sideband cooling, a quench laser coupling the metastable excited state $(|\uparrow\rangle)$ to a short-lived state is applied simultaneously with the RSB laser, effectively broadening the linewidth of the excited state to optimize the cooling rate $[46,48,61]$. In pulsed sideband cooling, the RSB and the quench (also called repump) lasers are applied sequentially $[49,62]$.

The RSB coupling strength depends strongly on the motional state $[58,63]$ and outside the Lamb-Dicke regime even exhibits points of vanishing coupling for certain initial motional states, effectively disabling further cooling beyond these points (see Fig. 2). While this regime has been studied theoretically [64-66], no experimental investigations are known to us.

Here we demonstrate fast and robust sympathetic groundstate cooling of a ${ }^{24} \mathrm{MgH}^{+}$molecular ion along one direction of motion using a ${ }^{25} \mathrm{Mg}^{+}$cooling ion. Starting with a 
(a)

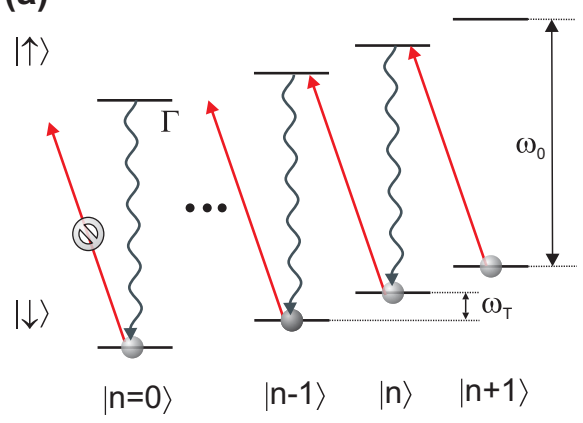

(b)

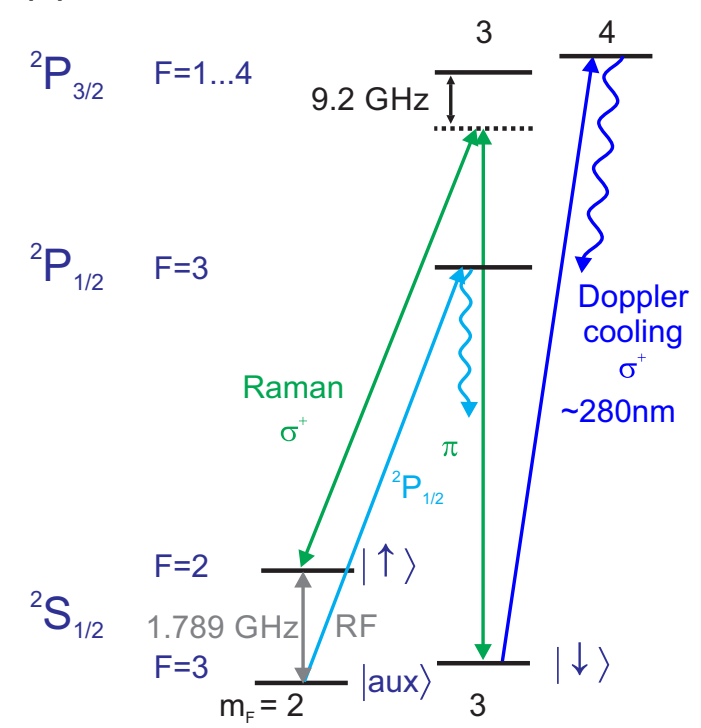

FIG. 1. (Color online) Principle of resolved sideband cooling and implementation in ${ }^{25} \mathrm{Mg}^{+}$. (a) Resolved SBC requires the linewidth $\Gamma$ of the transition to be smaller than the trap frequency $\omega_{\mathrm{T}}$, so that individual transitions can be selectively driven. With the cooling laser tuned to the first-order RSB, one quantum of motion is removed from the system in each absorption event. In the Lamb-Dicke regime, a change in motional state is suppressed upon decay back to the $|\downarrow\rangle$ state. (b) Relevant level structure for SBC of a ${ }^{25} \mathrm{Mg}^{+}$ion. Doppler cooling and Raman transitions are performed by coupling the ${ }^{2} S_{1 / 2}$ and ${ }^{2} P_{3 / 2}$ states. Compared to a previous implementation of SBC [67], a second laser is added for repumping the $\mid$ aux $\rangle$ state via the ${ }^{2} S_{1 / 2} \leftrightarrow$ ${ }^{2} P_{1 / 2}$ transition. $\mathrm{rf}$ radiation couples the states $|\uparrow\rangle$ and $|\mathrm{aux}\rangle$.

Doppler-cooled single trapped ${ }^{25} \mathrm{Mg}^{+}$ion, we investigate in Sec. II a novel repumping scheme for pulsed Raman sideband cooling, in which the excited electronic state in the cooling cycle is effectively quenched to the electronic ground state via coupling to a short-lived excited state. We demonstrate that the requirements on meeting the optimum RSB pulse length are significantly relaxed for the new quasicontinuous scheme compared to conventional pulsed Raman SBC. As a consequence of the large linewidth of the Doppler cooling transition in ${ }^{25} \mathrm{Mg}^{+}$, a significant amount of motional state population is trapped above the point of vanishing coupling strength for RSBs. We employ second-order RSB transitions to sweep the population beyond this point and demonstrate in Sec. IV that
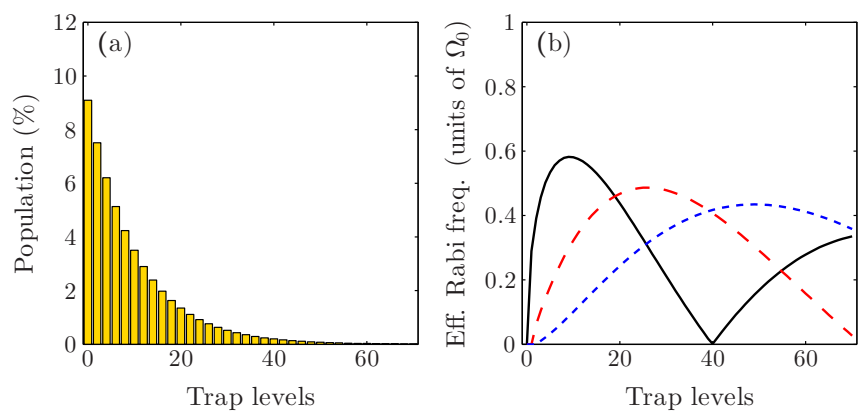

FIG. 2. (Color online) Motional state population and effective Rabi frequencies. (a) Typical population distribution over motional states after Doppler cooling, corresponding to $\bar{n} \approx 10$. (b) Effective Rabi frequencies as a function of the motional state calculated with a Lamb-Dicke parameter $\eta=0.30$ according to Eq. (1). The effective Rabi frequencies for different RSB orders have zero points at different motional excitations. Solid/dashed/dotted: first/second/third-order RSB.

ground-state cooling with Lamb-Dicke factors as large as 0.45 and motional level populations up to $n \sim 120$ become possible by employing RSBs up to eighth order. By optimizing the ground-state cooling rate in terms of pulse lengths for firstand second-order RSB and the time spent on cooling with each sideband order, a total cooling time as short as $500 \mu \mathrm{s}$ for cooling a single ion from Doppler temperature $(\bar{n} \sim 10)$ to the motional ground state is demonstrated. The experimental results are supported by numerical master equation simulations of the system (Appendix). In Sec. III this scheme is extended to cool a ${ }^{24} \mathrm{MgH}^{+} /{ }^{25} \mathrm{Mg}^{+}$ion crystal to the ground state by interleaved cooling of both axial modes. After optimizing the RSB pulse length for each mode and the time spent on cooling each, a total cooling time of $2.5 \mathrm{~ms}$ is achieved, resulting in a mean residual motional excitation of 0.06(3) and 0.03(3) for the in- and out-of-phase mode, respectively.

\section{SBC OF A SINGLE ION}

\section{A. Experimental setup}

The current work is based on previous results [67], where we demonstrated a pulsed Raman sideband cooling scheme using a single laser system for ${ }^{25} \mathrm{Mg}^{+}$. In brief, a ${ }^{25} \mathrm{Mg}^{+}$ ion is trapped via isotope-selective photoionization in a linear Paul trap with axial and radial motional frequencies of 2.21 and $\sim 5 \mathrm{MHz}$, respectively. The relevant levels for SBC of a single ${ }^{25} \mathrm{Mg}^{+}$ion are shown in Fig. 1(b). A frequency-quadrupled fiber laser $\left({ }^{2} P_{3 / 2}\right.$ laser in the following) provides the radiation for Doppler cooling, coherent control, and state detection on the ${ }^{25} \mathrm{Mg}^{+}$ion. Doppler cooling on the $\left.\left.\right|^{2} S_{1 / 2}, F=3, m_{F}=3\right\rangle\left.\equiv|\downarrow\rangle \leftrightarrow\right|^{2} P_{3 / 2}, F=4, m_{F}=$ 4) transition for $400 \mu$ s yields a $\bar{n} \sim 10$ as the starting point for the ground-state cooling sequence. Coherent electronic and motional state manipulation is implemented via Raman transitions, coupling the $|\downarrow\rangle$ and $|\uparrow\rangle \equiv\left|{ }^{2} S_{1 / 2}, F=2, m_{F}=2\right\rangle$ states. No change in the motional state correspond to carrier (CAR) transitions, while addition of phonons correspond to blue sideband (BSB) transitions upon changing the electronic state from $|\downarrow\rangle$ to $|\uparrow\rangle$. The Raman beams are generated via 
acousto-optic modulators from the ${ }^{2} P_{3 / 2}$ laser and are detuned with respect to the ${ }^{2} P_{3 / 2}$ state by around $9.2 \mathrm{GHz}$. The beams have $\pi$ and $\sigma^{+}$polarization to maximize the coupling strength and are at right angles to each other with the difference wave vector aligned along the axial direction of the trap. This results in a Lamb-Dicke parameter of $\eta=0.3$ [67] for the axial mode of interest here. The limited detuning of the Raman laser beams leads to off-resonant excitations, which result in dephasing and population loss during coherent manipulation as further discussed in Sec. II D. This effect limits the detection fidelity of the motional-ground-state population and the final $\bar{n}$ detectable after cooling. It can be reduced by employing a separate Raman laser system with larger detuning.

In the previously implemented SBC scheme [67] the Rabi frequency $\Omega_{n^{\prime}, n}$ of the Raman transition between motional state $|n\rangle$ and $\left|n^{\prime}\right\rangle$ was calculated according to $[58,63]$

$$
\Omega_{n^{\prime}, n}=\Omega_{0} \exp \left(\frac{-\eta^{2}}{2}\right) \sqrt{\frac{n_{<} !}{n_{>} !}} \eta^{\left|n^{\prime}-n\right|} L_{n_{>}}^{\left|n^{\prime}-n\right|}\left(\eta^{2}\right),
$$

where $\Omega_{0}$ is the CAR Rabi frequency, $n_{<}\left(n_{>}\right)$denotes the smaller (larger) of $n, n^{\prime}$, and $L_{n}^{a}(x)$ are the generalized Laguerre polynomials. This allowed the determination of the $\pi$ time for complete population transfer on the first- and second-order RSBs (see Fig. 2). This way, RSB $\pi$ pulses were applied to sweep the population starting from $n=40$ to the motional ground state. After each RSB pulse, multiple repump cycles involving optical excitation of the $|\uparrow\rangle \rightarrow{ }^{2} P_{3 / 2}$ transition and RF state inversion pulses between $|\mathrm{aux}\rangle$ and $|\uparrow\rangle$ are necessary to transfer the population back to the $|\downarrow\rangle$ state, since the excited state in ${ }^{2} P_{3 / 2}$ can also decay into the $\mid$ aux $\left.\rangle\left.\equiv\right|^{2} S_{1 / 2}, F=3, m_{F}=2\right\rangle$ state. The cooling speed of this implementation was mainly limited by the radio-frequency (rf) coupling strength and the required number of sweeps (2-3) to reach the ground state.

\section{B. Cooling scheme}

In the approach described in the following, we significantly reduce the cooling time by adding a dedicated repump laser for faster repumping of the $\mid$ aux $\rangle$ state via the $\left.\right|^{2} P_{1 / 2}, F=3, m_{F}=$ 3) state and by using RSB pulses with equal length. The experimental sequence starts with Doppler cooling. For SBC we first apply a series of second-order RSB pulses followed by a series of first-order RSB pulses of fixed lengths. After each SBC pulse a short $\left(t_{\mathrm{r}}=3 \mu \mathrm{s}\right)$ optical repumping pulse using the $\sigma^{+}$beam of the Raman laser tuned to resonance and the ${ }^{2} P_{1 / 2}$ laser is applied to clear out the population left in $|\operatorname{aux}\rangle$ and $|\uparrow\rangle$. In the experiment the actual elapsed time between two SBC pulses equals $t_{\mathrm{r}}^{\prime}=5 \mu$ s caused by delays from the control electronics. In addition, we can use the ${ }^{2} P_{1 / 2}$ laser and the rf coupling between $|\mathrm{aux}\rangle$ and $|\uparrow\rangle$ to implement a quench coupling during the Raman RSB pulses. This quench coupling opens up an additional decay channel for the $|\uparrow\rangle$ state back to the ground state $|\downarrow\rangle$ with a controllable decay rate, implementing a fusion between pulsed and continuous sideband cooling. After SBC, the population in the motionally excited states is probed by driving a rf $\pi$ pulse to transfer all population from $|\downarrow\rangle$ to $|\uparrow\rangle$, followed by a stimulated Raman adiabatic passage (STIRAP) pulse on the first blue

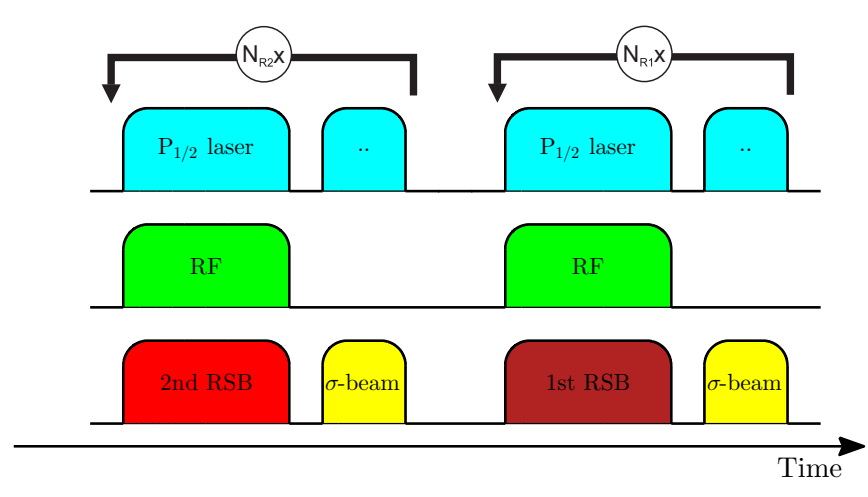

FIG. 3. (Color online) Sequence for single ion sideband cooling. Sequence for SBC a single ${ }^{25} \mathrm{Mg}^{+}$. The sequence starts by repeating $N_{\mathrm{R} 2}$ second-order RSBs, followed by $N_{\mathrm{R} 1}$ first-order RSBs with fixed pulse lengths. After each SBC pulse, repumping pulses consisting of ${ }^{2} P_{1 / 2}$ and $\sigma$ beams are applied to bring the ion back to $|\downarrow\rangle$ state.

sideband (BSB) [68]. This maps motionally excited states $(n>0)$ onto the state $|\downarrow\rangle$, while the motional-ground-state population $(n=0)$ remains in the state $|\uparrow\rangle$ [69].

We optimize the cooling rate for a fixed total cooling time $T_{\mathrm{c}}$ by changing the amount of time spent on cooling via secondand first-order RSBs, characterized by a time scaling factor $\alpha$. Depending on the pulse length of the first- (second)-order RSB pulses $t_{\mathrm{R} 1}\left(t_{\mathrm{R} 2}\right)$, we apply $N_{\mathrm{R} 2}$ second-order and $N_{\mathrm{R} 1}$ first-order RSB pulses

$$
\begin{aligned}
& N_{\mathrm{R} 2}=\left\lfloor\frac{\alpha T_{\mathrm{c}}}{t_{\mathrm{R} 2}}\right\rfloor, \\
& N_{\mathrm{R} 1}=\left\lfloor\frac{(1-\alpha) T_{\mathrm{c}}}{t_{\mathrm{R} 1}}\right\rfloor,
\end{aligned}
$$

where the sign $\lfloor x\rfloor=\max \{n \in \mathbb{Z}, n \leqslant x\}$ denotes the floor function. Short padding pulses are added to keep $T_{\mathrm{c}}$ fixed. The total time spent on ground-state cooling during the cooling sequence as shown in Fig. 3 is expressed as

$$
T_{\text {total }}=T_{\mathrm{c}}+N_{\mathrm{r}} t_{\mathrm{r}}^{\prime}
$$

where $N_{\mathrm{r}}$ is the total number of repump pulses.

Since we are interested in the cooling time, we investigate the dynamics of SBC by probing the population in the motional ground state with the STIRAP sideband pulse for different SBC times $T_{\mathrm{c}}$ instead of optimizing for lowest $\bar{n}$. Assuming a constant cooling rate $W$ during the SBC cycle, the mean occupation of the motional states decays exponentially [70] as

$$
\bar{n}(t)=\bar{n}_{\mathrm{f}}\left(1-e^{-W t}\right)+\bar{n}_{\mathrm{i}} e^{-W t},
$$

where $\bar{n}_{\mathrm{i}}$ and $\bar{n}_{\mathrm{f}}$ are the initial and final mean occupation, respectively, and $W$ is the cooling rate. Assuming a thermal distribution with mean occupation of $\bar{n}(t)$ after SBC for a duration $t$, the population in the motional ground state can be expressed as

$$
\begin{aligned}
P_{0}(t) & =\frac{1}{1+\bar{n}_{\mathrm{f}}\left(1-e^{-W t}\right)+\bar{n}_{\mathrm{i}} e^{-W t}} \\
& =\frac{1}{1+\bar{n}_{\mathrm{f}}+\left(\bar{n}_{\mathrm{i}}-\bar{n}_{\mathrm{f}}\right) e^{-t / T_{0}}} .
\end{aligned}
$$


The desired cooling time constants $T_{0}=1 / \mathrm{W}$ for sets of parameters $\left(t_{\mathrm{R} 1}, t_{\mathrm{R} 2}, \alpha\right)$ are extracted by fitting data to this model function in which only one parameter is changed and $\bar{n}_{\mathrm{i}}$ and $\bar{n}_{\mathrm{f}}$ are common fit parameters. By choosing $T_{\mathrm{c}} \sim 7 T_{0}$, we ensure reaching the steady state.

\section{Optimization of experimental parameters}

Using the procedure described in the previous section, we vary the pulse length of the RSB pulses, the time scaling factor $\alpha$, and the optical power of the ${ }^{2} P_{1 / 2}$ repump laser to optimize the cooling rate. Furthermore, we explore the transition between pulsed and quench cooling by adding the ${ }^{2} P_{1 / 2}$ repump laser and $\mathrm{rf}$ coupling between the $|\mathrm{aux}\rangle$ and $|\uparrow\rangle$ states during the RSB pulses. A numerical simulation based on optical Bloch equations described in the Appendix supports our findings quantitatively.

\section{Pulse length optimization}

We first optimize the cooling time constant as a function of the pulse length for the first- and second-order RSB pulses. For each parameter set, a scan of the SBC time $T_{\mathrm{c}}$ is performed, which allows us to derive the corresponding cooling time constant as shown in Fig. 4(d). In Figs. 4(a) and 4(b) the experimentally determined cooling time constants $T_{0}$ are plotted against the pulse lengths. The shortest $T_{0}$ is
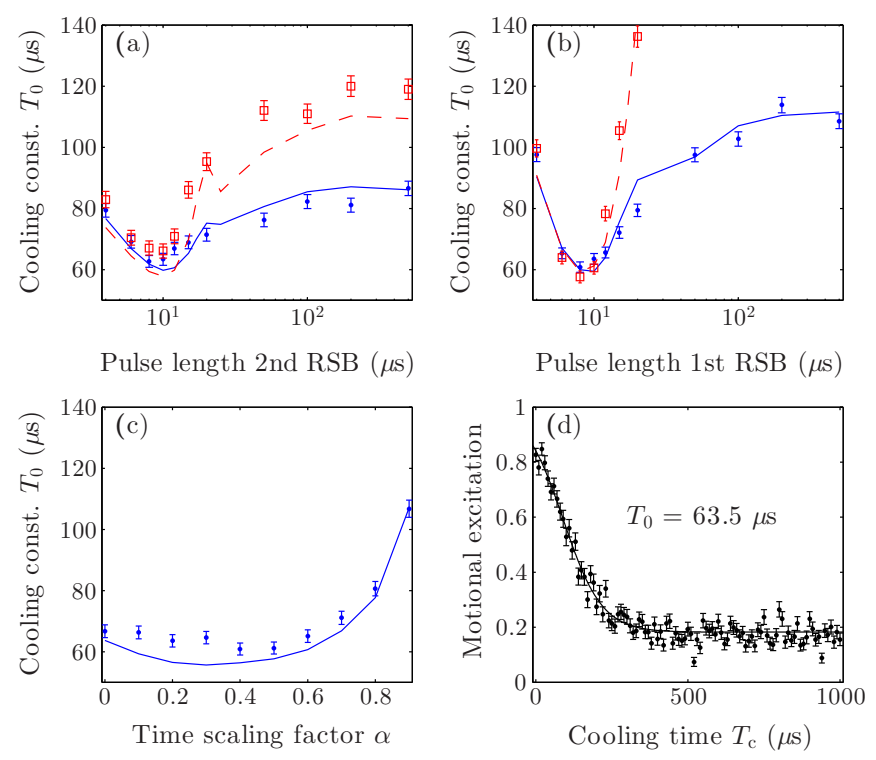

FIG. 4. (Color online) Dependence of the cooling time constants on experimental parameters. Cooling time constants as a function of the pulse lengths of the (a) second-order and (b) first-order RSB pulses with $\alpha=0.5$ while fixing the nonscanned pulse length near its optimal value. Blue point (red square): Experimentally determined cooling constants with the quench coupling on (off) during the RSB pulse. The quench coupling induces an effective decay rate of $42 \mathrm{~ms}^{-1}$ from the $|\uparrow\rangle$ state to the $|\downarrow\rangle$ state. (c) Cooling time constant as a function of the time scaling factor with $t_{\mathrm{R} 2}=10 \mu \mathrm{s}$ and $t_{\mathrm{R} 1}=10 \mu \mathrm{s}$. The lines in (a)-(c) are the result of master equation simulations using the experimental parameters. (d) Residual motional excitation as a function of the total cooling time $T_{\mathrm{c}}$. The line is the fit of Eq. (5) to the experimental data, which gives the cooling constant $T_{0}$. obtained for first- and second-order RSB pulse lengths of around $10 \mu \mathrm{s}$. The experimentally determined $\pi$ time for a RSB pulse starting at $|n=1\rangle$ is $T_{\pi} \approx 16 \mu \mathrm{s}$. Averaging over the $\pi$ times for first- and second-order RSBs according to Eq. (1), weighted by the thermal occupation of the initial states, limited for each sideband to the states where the coupling strength of the respective sideband dominates, gives for both sidebands average $\pi$ times of $\sim 10 \mu$ s in agreement with the experimentally optimized values. The fact that a constant $\pi$ time is sufficient for efficient cooling is a consequence of the relatively small variation of the Rabi frequencies in the relevant range of motional levels as can be seen from Fig. 2.

In standard pulsed Raman SBC, the cooling time constant strongly rises upon deviation from the optimum pulse lengths [red points and dashed lines in Figs. 4(a) and 4(b)]. This dependence becomes much weaker for long pulses [blue points and solid lines in Figs. 4(a) and 4(b)] by applying the quench coupling during the RSB pulses which opens a decay channel for the $|\uparrow\rangle$ state. In the experiment, this decay rate was adjusted to about $42 \mathrm{~ms}^{-1}$ or a decay constant of about $24 \mu \mathrm{s}$ to achieve the highest cooling rate. At this decay rate, the effect of repumping is negligible for pulses shorter than the optimum pulse length and the scheme is equivalent to standard pulsed SBC. For RSB pulses longer than the optimum pulse length, the ion in the $|\uparrow\rangle$ state decays back to the $|\downarrow\rangle$ state through the new channel. The ion cycles between the $|\downarrow\rangle$ and the $|\uparrow\rangle$ states and therefore more than one phonon can be removed within a single RSB pulse. The scheme thus becomes equivalent to continuous SBC. This quasicontinuous cooling scheme is insensitive to the exact pulse length of the RSB pulses and provides high robustness against intensity/pointing fluctuation of the Raman lasers.

\section{Time scaling factor optimization}

The time scaling factor $\alpha$ as defined in Eq. (2) distributes the SBC time $T_{\mathrm{c}}$ into the time spent on the second-order RSB $T_{\mathrm{R} 2}=\alpha T_{\mathrm{c}}$ and on the first-order RSB $T_{\mathrm{R} 1}=(1-\alpha) T_{\mathrm{c}}$. Due to the dependence of the Rabi frequency on the motional quantum number [see Fig. 2 and Eq. (1)], the second-order RSB pulses are more efficient in cooling the population in motional states $n>20$ at the starting stage of the SBC cycle. For the lower motional states, the first-order RSB pulses become more efficient, so that an $\alpha$ that is too large also increases the SBC cooling time constant [Fig. 4(c)]. Since the variation of first- and second-order RSB Rabi frequencies with $n$ are very slow, the cooling time spent on either order only significantly influences the cooling time constant for the extreme cases of $\alpha \rightarrow 0$ and $\alpha \rightarrow 1$ and remains otherwise flat.

\section{Cooling results}

The final $\bar{n}$ after SBC is extracted from the red and blue sideband excitation. Assuming that the ion stays in a thermal distribution after SBC, the ratio of excitations on the first red and blue sideband fulfills the following relation [71]:

$$
Q:=\frac{\rho^{\mathrm{RSB}}(t)}{\rho^{\mathrm{BSB}}(t)}=\frac{\bar{n}}{1+\bar{n}},
$$

where $\rho^{\mathrm{RSB}}(t)$ and $\rho^{\mathrm{BSB}}(t)$ are the excitation probabilities on the first RSB and BSB at time $t$, respectively. For this analysis we subtracted a constant signal background from the data, 


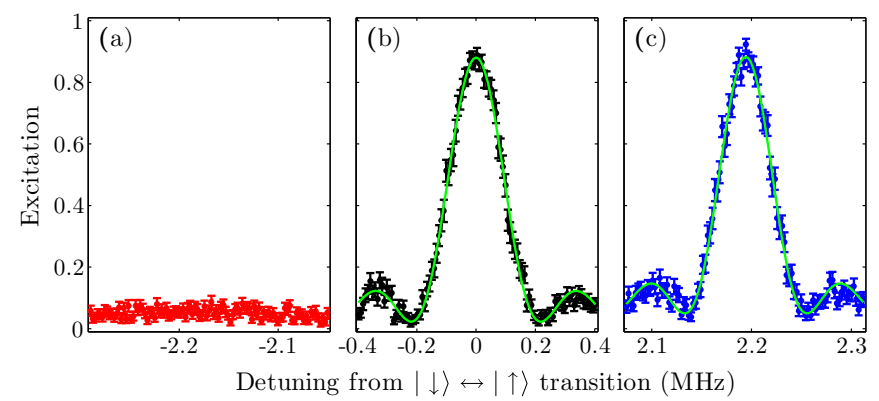

FIG. 5. (Color online) Rabi excitation of ${ }^{25} \mathrm{Mg}^{+}$after sideband cooling. (a)-(c) Frequency scans of Raman transitions over first RSB (red), carrier (black), and first BSB (blue) transitions after SBC of a single ${ }^{25} \mathrm{Mg}^{+}$.

which arises from off-resonant Raman excitation and thus does not affect the determined temperature. With the SBC sequence described in Sec. II B, we achieve $\bar{n}=\rho^{\mathrm{RSB}} /\left(\rho^{\mathrm{BSB}}-\rho^{\mathrm{RSB}}\right) \approx$ 0.01(2) as shown in Figs. 5(a)-5(c) after a cooling time of $T_{\mathrm{c}}=500 \mu \mathrm{s}$. This represents a reduction in cooling time by more than a factor of 30 compared to Ref. [67].

\section{SYMPATHETIC GROUND-STATE COOLING OF A MOLECULAR ION}

In this section we adapt the quasicontinuous cooling scheme for sympathetic cooling of a molecular ion using ${ }^{25} \mathrm{Mg}^{+}$as the cooling ion species.

\section{A. Loading and sympathetic Doppler cooling of ${ }^{24} \mathrm{MgH}^{+}$}

A two-ion crystal consisting of a ${ }^{25} \mathrm{Mg}^{+}$and a ${ }^{24} \mathrm{MgH}^{+}$ion is prepared by isotope-selective photoionization loading [72] of a pair of ${ }^{25} \mathrm{Mg}^{+}$and ${ }^{24} \mathrm{Mg}^{+}$ions with the Doppler cooling laser tuned near the resonance of the ${ }^{25} \mathrm{Mg}^{+}$cooling transition. Then, the laser is tuned near to the cooling resonance of ${ }^{24} \mathrm{Mg}^{+}$and hydrogen gas is leaked into the vacuum system through a leak valve, increasing the pressure up to $\sim 5 \times 10^{-9}$ mbar. After a photochemical reaction during which the excited ${ }^{24} \mathrm{Mg}^{+}$ion reacts with a hydrogen molecule to form ${ }^{24} \mathrm{MgH}^{+}$[73], the fluorescence of the ${ }^{24} \mathrm{Mg}^{+}$ion vanishes. After closing the leak valve and tuning the laser back to the ${ }^{25} \mathrm{Mg}^{+}$resonance, cooling commences and the mass of the dark ion is determined via mass spectrometry using parametric heating of the two-ion crystal [74]. Since the mass of the two ions is almost identical, we do not expect significant deviations from Doppler cooling temperature, even in the presence of additional heating [75].

\section{B. Two-mode sympathetic ground-state cooling sequence}

For sympathetic ground-state cooling of the molecular ion we use a slightly modified pulse sequence compared to Sec. II B. In contrast to the case of a single ${ }^{25} \mathrm{Mg}^{+}$, the motion of the ions is described by two modes along the axial direction, the in-phase (IP) and the out-of-phase (OP) mode with secular frequencies of $\omega_{\mathrm{T}}^{\mathrm{IP}}=2 \pi \times 2.21 \mathrm{MHz}$ and $\omega_{\mathrm{T}}^{\mathrm{OP}}=$ $2 \pi \times 3.85 \mathrm{MHz}$. The Lamb-Dicke parameters for the coupling of the Raman lasers to the ${ }^{25} \mathrm{Mg}^{+}$ion are $\eta_{\mathrm{IP}}=0.21$ and $\eta_{\mathrm{OP}}=0.16$. With these Lamb-Dicke parameters, the effective

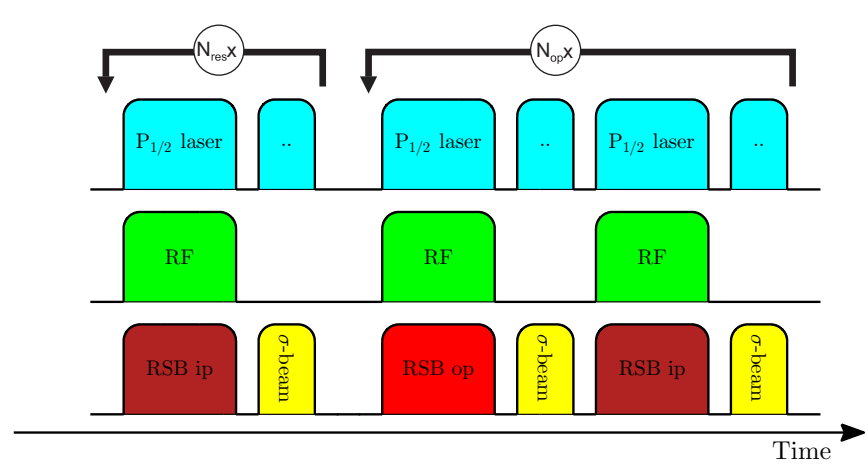

FIG. 6. (Color online) Sequence for sympathetic sideband cooling of a two-ion crystal. The in-phase and out-of-phase axial modes of a ${ }^{25} \mathrm{Mg}^{+} /{ }^{24} \mathrm{MgH}^{+}$two-ion crystal are cooled in an interleaved fashion to ensure simultaneous cooling.

Rabi frequencies show no zero crossings over the range of trap levels with significant population after Doppler cooling, so no higher-order RSB pulses are necessary. However, with Lamb-Dicke factors as large as these, cooling of a single mode is not sufficient to enable high fidelity operations involving only this mode. The other mode acts as a spectator mode which modifies the effective Rabi frequency of the mode of interest depending on its motional state [63]. Therefore, we have implemented an interleaved pulse sequence for SBC both axial modes simultaneously as shown in Fig. 6. Depending on the time scaling factor $\alpha^{\prime}$, which in this case distributes the total SBC time $T_{\mathrm{c}}$ into time spent for RSB on the IP mode $\left(1-\alpha^{\prime}\right) T_{\mathrm{c}}$ and time for RSB on the OP mode $\alpha^{\prime} T_{\mathrm{c}}$, we apply

$$
\begin{gathered}
N_{\mathrm{IP}}=\left\lfloor\frac{\left(1-\alpha^{\prime}\right) T_{\mathrm{c}}}{t_{\mathrm{IP}}}\right\rfloor, \\
N_{\mathrm{OP}}=\left\lfloor\frac{\alpha^{\prime} T_{\mathrm{c}}}{t_{\mathrm{OP}}}\right\rfloor
\end{gathered}
$$

pulses on the IP and the OP mode, respectively. Here $t_{\mathrm{IP}}, t_{\mathrm{OP}}$ are the pulse lengths of the RSB pulses on the IP and the OP mode. In the case $N_{\mathrm{IP}}>N_{\mathrm{OP}}\left(N_{\mathrm{IP}}<N_{\mathrm{OP}}\right)$ we start the SBC cycle with $N_{\text {res }}=\left|N_{\text {IP }}-N_{\text {OP }}\right|$ pulses on the IP (OP) mode, followed by $N_{\mathrm{OP}}\left(N_{\mathrm{IP}}\right)$ pulses on the IP and the OP mode in an interleaved fashion. After every single SBC pulse, a repumping pulse as described above is applied to clear out the $|\uparrow\rangle$ and $\mid$ aux $\rangle$ states in addition to the quench coupling present also during the RSB pulses. Optimizations similar to the case of a single ${ }^{25} \mathrm{Mg}^{+}$are performed to minimize the total duration of SBC.

\section{Pulse length optimization}

The length of the RSB pulse on the IP (OP) mode is optimized by fixing the other mode's RSB pulse length. As described in Sec. II B, the cooling time constant is derived from the motional-ground-state population measured by performing a STIRAP pulse resonant with the first BSB of either mode for different cooling times $T_{\mathrm{c}}$. We spent an equal amount of time for cooling on both modes $\left(\alpha^{\prime}=0.5\right)$ and ensure near steady-state conditions by performing cooling up to $T_{\mathrm{c}} \approx 2.5 \mathrm{~ms}$. The highest cooling rate is observed with a pulse length of $15 \mu \mathrm{s}(20 \mu \mathrm{s})$ for the RSB on the IP (OP) mode 

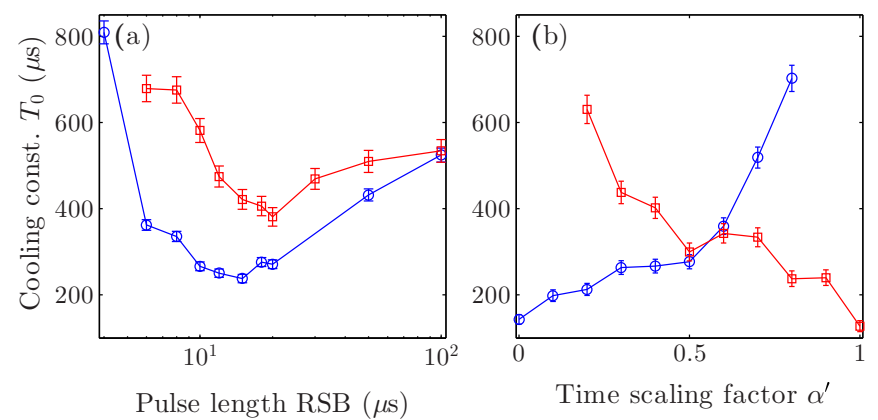

FIG. 7. (Color online) Dependence of the cooling time constant on experimental parameters for sympathetic sideband cooling a twoion crystal. (a) The cooling time constant as a function of the optimal pulse length of the Raman RSB pulses on the IP (blue circles) and OP (red squares) mode. (b) The cooling constant as a function of the optimal time scaling factor $\alpha^{\prime}$. The lines connect the points and are guides to the eye.

as shown in Fig. 7(a). With a $\pi$ time of $T_{\pi}^{(\mathrm{IP})} \approx 21 \mu \mathrm{s}$ and $T_{\pi}^{(\mathrm{OP})} \approx 26.5 \mu$ s for the first RSB starting in the $|n=1\rangle$ state, the Rabi frequency according to Eq. (1) averaged over the thermal occupation of motional states results in a mean $\pi$ time of 14 and $25 \mu$ s for the IP and OP mode, respectively.

As in the single-ion case discussed in Sec. II C, the increase in the cooling time constant for pulse lengths longer than the optimum pulse length is rather mild as a consequence of the quench coupling during RSB pulses.

\section{Time scaling factor optimization}

The time scaling factor in the SBC sequence for cooling a two-ion crystal divides the total cooling time $T_{\mathrm{c}}$ into the time spent on cooling the IP mode and the OP mode for the optimized pulse lengths derived from the measurements in the previous section. The measured SBC cooling time constants for the two modes as a function of $\alpha^{\prime}$ are shown in Fig. 7(b). Although an $\alpha^{\prime}$ that is too small (large) accelerates the cooling of the IP (OP) mode, the cooling time of the opposite mode increases. The optimal value for the time scaling factor $\alpha^{\prime} \approx 0.5$ is determined by the point where SBC of both the IP and OP mode are achieved with the shortest total duration of the SBC cycle. Interestingly, this corresponds to a different number of RSB pulses for the two modes.

\section{E. Cooling results}

Figure 8 shows frequency scans of the carrier and both RSB and BSB transitions after SBC the IP and OP modes along the axial direction with optimized parameters. The final $\bar{n}$ is determined from the red and blue sideband excitations of each mode as described in Sec. II D. As before, the offset from off-resonant Raman excitations has been subtracted for the analysis. We reach a $\bar{n}_{\mathrm{IP}}=0.06(3)$ for the IP mode and $\bar{n}_{\mathrm{OP}}=$ 0.03(3) for the OP mode after a total cooling time $T_{\text {total }} \sim$ $2.5 \mathrm{~ms}$. Compared to an extension of the SBC scheme described in Ref. [67] to two-mode ground-state cooling, the optimized new scheme reduces the cooling time by a factor of 8 .

\section{SIDEBAND COOLING BEYOND THE LAMB-DICKE REGIME}

For some applications large Lamb-Dicke factors are desirable, since they enhance the sensitivity of the ion's motion to small forces. This is the case for photon recoil spectroscopy [11] or the nondestructive internal state detection of a molecular ion using oscillating dipole forces [76-78]. In the following we extend the single-ion ground-state cooling scheme presented in Sec. II to enable efficient ground-state cooling outside the Lamb-Dicke regime, which is readily extended to the multi-ion case discussed Sec. III.

At a trap frequency of $1 \mathrm{MHz}$ and a temperature of $1 \mathrm{mK}$ theoretically achievable with Doppler cooling a ${ }^{25} \mathrm{Mg}^{+}$ion, states up to $n \sim 120$ are significantly populated, leaving less than $0.3 \%$ population in levels $n>120$ [Fig. 9(a)]. At this trap frequency, the Lamb-Dicke parameter in our system is $\eta=0.45$. The effective Rabi frequency for RSB transitions depends strongly on the trap levels and shows several points of vanishing coupling over the range of trap levels with significant population [see Fig. 9(b) and Eq. (1)]. Employing pulses on the second-order sideband as in the scheme for a single ${ }^{25} \mathrm{Mg}^{+}$is no longer sufficient. Instead, we employ as many higher-order sidebands as necessary. For larger motional states, successively higher-order sidebands exhibit a maximum in their coupling rate. Moreover, pulses on higher-order sidebands are more efficient since more than one phonon is removed per sideband pulse. For simplicity we split the total cooling time $T_{\mathrm{c}}$ equally
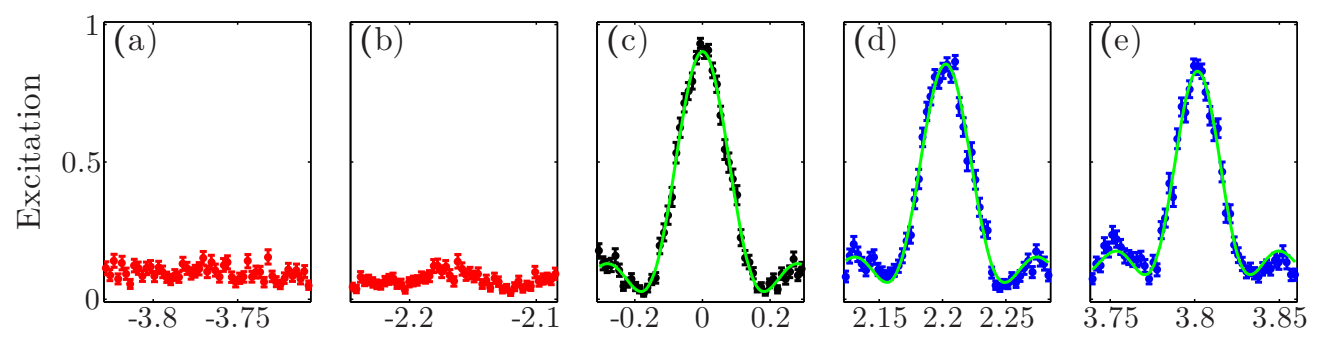

Detuning from $|\downarrow\rangle \leftrightarrow|\uparrow\rangle$ transition $(\mathrm{MHz})$

FIG. 8. (Color online) Rabi excitation of ${ }^{25} \mathrm{Mg}^{+}$after two-mode $\mathrm{SBC}$ a ${ }^{24} \mathrm{MgH}^{+} /{ }^{25} \mathrm{Mg}^{+}$crystal. Frequency scans of Raman transitions over the first RSB (red) of the (a) OP and (b) IP modes, (c) the carrier (black), and the first BSB (blue) (d) IP and (e) OP modes after SBC both modes on ${ }^{25} \mathrm{Mg}^{+}$. 

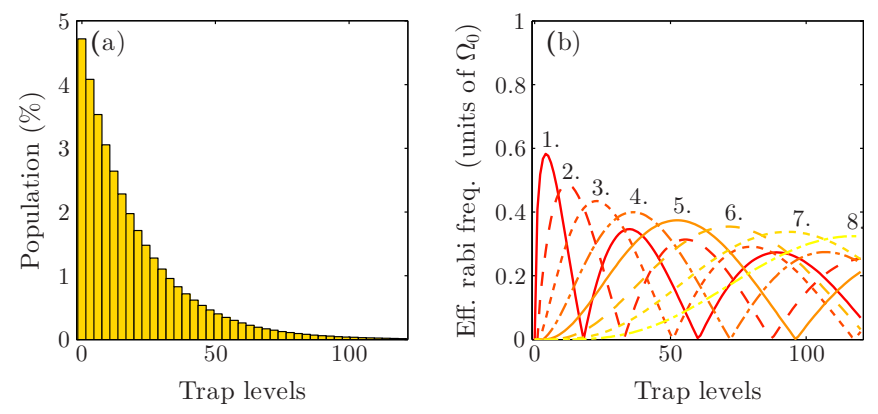

FIG. 9. (Color online) Motional state population and effective Rabi frequency for small trap frequency. (a) Distribution of the motional levels at a motional frequency of $\omega_{\mathrm{T}}=1 \mathrm{MHz}$ and a temperature of $T=1 \mathrm{mK}$ corresponding to the Doppler cooling limit of the ${ }^{25} \mathrm{Mg}^{+}$ion. Less than $0.3 \%$ of the population are in the trap levels $|n>120\rangle$. (b) Effective Rabi frequencies at $\eta=0.45$ for different RSB orders are shown as a function of the trap levels. For ions located in higher trap levels, RSB pulses of higher-orders $\beta(n)$ are more efficient for cooling.

between all the sideband orders and apply to all of them the same pulse length, thus extending the sequence shown in Fig. 3 to higher-order modes. This is a valid approach, since the variation in maximum Rabi frequency across the higher-order sidebands is small. Simultaneously to each RSB pulse, we apply the quench coupling to further reduce the sensitivity to the optimum pulse length. After each RSB pulse, a repumping pulse as described before is applied. The optimal number of applied sideband orders, derived from measurements of the cooling time constant as a function of the maximally applied sideband order, is shown in Fig. 10(a). In the optimal sequence we need to apply sideband pulses up to the eighth order, which confirms the prediction of Fig. 9(b).

More generally, with known Lamb-Dicke parameter and thermal distribution over motional states after Doppler cooling, a corresponding SBC strategy can be used to achieve the most efficient cooling as illustrated in Fig. 10(b), where we define
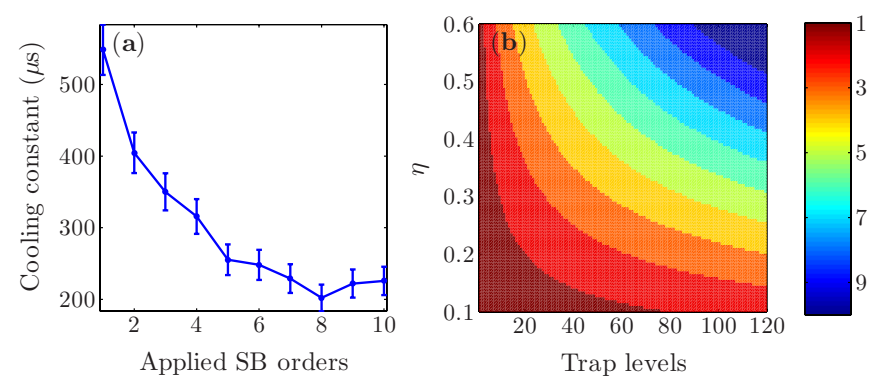

FIG. 10. (Color online) Determination of the maximum sideband order. (a) The cooling time constant as a function of applied sideband orders. The optimal number for the sideband orders determined experimentally confirms the prediction shown in Fig. 9(b). (b) RSB orders with highest effective cooling rate as a function of the trap level and the Lamb-Dicke parameter. With known Lamb-Dicke parameter and motional distribution a corresponding SBC sequence can be adopted. the cooling efficiency as $\beta(n) \Omega_{n-\beta, n}$. For a selected upper trap level to be addressed by cooling and a known Lamb-Dicke parameter, the sideband order with highest cooling efficiency is plotted. This strategy can be combined with multimode cooling through interleaved pulse sequences as shown in Sec. III.

\section{SUMMARY AND DISCUSSION}

A quasicontinuous sideband cooling scheme was introduced by applying a quench coupling to the excited state in pulsed Raman sideband cooling. For long Raman pulses this scheme allows multiple RSB-spontaneous emission cycles, reminiscent of continuous quench cooling. This feature makes SBC more robust and significantly reduces the optimization required for optimum cooling, since RSB pulses that are longer than the $\pi$ time for a specific initial motional state contribute more efficiently to cooling. Using this scheme, we performed an optimization of the cooling rate of single-ion ground-state cooling and two-ion sympathetic ground-state cooling, significantly outperforming previous results [67]. These findings are important to minimize the time required for ground-state cooling, thus reducing overhead in experiments with trapped ions. The results are applicable to other commonly used hyperfine qubit systems such as ${ }^{9} \mathrm{Be}^{+},{ }^{111} \mathrm{Cd}^{+}$, or ${ }^{171} \mathrm{Yb}^{+}$. A variation of the scheme can be applied to other systems like ${ }^{40} \mathrm{Ca}^{+},{ }^{24} \mathrm{Mg}^{+}$in high magnetic field or optically trapped neutral atoms.

We have also demonstrated experimentally that SBC to the ground state is possible outside the Lamb-Dicke regime by employing higher-order RSB transitions, thus confirming theoretical predictions [64-66]. This regime is particularly relevant for systems where the Doppler cooling linewidth significantly exceeds the motional frequency as is the case for optically trapped neutral atoms, or in situations where the Lamb-Dicke factor is deliberately chosen as large as possible. This is the case for example in experiments which aim to detect small forces through the excitation of the ion's motion from the ground state, such as in photon recoil spectroscopy [11] and the detection of electric fields [62,79].

The demonstrated motional-ground-state cooling of a molecular ion, in particular when combined with cooling at low trap frequency, represents an important step towards the implementation of nondestructive state preparation and detection techniques [76-78] based on the detection of small optically induced and state-selective displacement forces acting on a trapped molecular ion.

\section{ACKNOWLEDGMENTS}

We acknowledge the support of DFG through QUEST and Grant SCHM2678/3-1. Y.W. acknowledges support from the Braunschweig International Graduate School of Metrology (BIGSM). We thank R. Blatt for generous loan of equipment.

\section{APPENDIX: NUMERICAL SIMULATION FOR SBC}

The dynamics of the system during quasicontinuous $\mathrm{SBC}$ is modeled using optical Bloch equations, where two electronic states $|\uparrow\rangle$ and $|\downarrow\rangle$ and 80 trap levels (Fig. 11) are considered. 


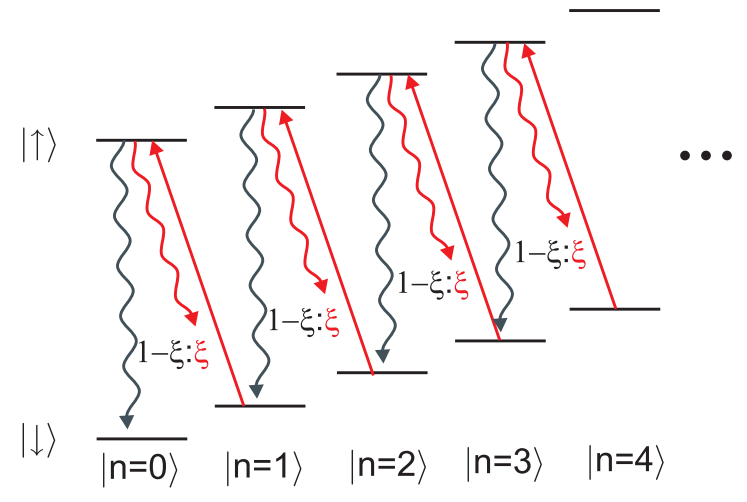

FIG. 11. (Color online) Relevant levels for numerical simulation of the dynamics during the Raman SBC cycles. The Raman lasers are tuned to be resonant with the $\beta$-order RSB ( $\beta=1$ in graph). Through the dissipative channels either from off-resonant excitation or via a repumping process followed by spontaneous emission, the ion is reinitialized to the $|\downarrow\rangle$ with a decay rate of 0.005 and $0.47 \mathrm{~s}^{-1}$, respectively. Spontaneous emission happens on the carrier transition with a probability $1-\xi$ and the remaining small fraction $\xi$ happens on the RSB transitions.

The Raman RSB pulses are included as a resonant coupling between $|\downarrow, n\rangle$ and $|\uparrow, n-\beta\rangle$ with $\beta$ indicating the sideband order used. The spontaneous decay during the Raman cooling cycle via the auxiliary states [80] is implemented with an effective decay rate $\Gamma_{\text {eff }}$ determined experimentally. This approach neglects effects from the actual multilevel electronic structure of ${ }^{25} \mathrm{Mg}^{+}$. Repumping after application of the RSB is implemented through a projection of the population onto the electronic ground state $|\downarrow\rangle$. The spatially averaged Lamb-Dicke factor for spontaneous emission along the axial direction is $\tilde{\eta}=0.134$. Heating from spontaneous emission is taken into account through a branching ratio of $1-\xi: \xi$ for emission on the CAR and RSB transition. Here $\xi$ is on the order of $3 \widetilde{\eta}^{2} \approx 0.05$, where the factor 3 considers the multiple scattering events until the ion falls into the $|\downarrow\rangle$ state. Combining all the ingredients above, we end up with the following optical
Bloch equations:

$$
\begin{aligned}
& \dot{\rho}_{\downarrow n, \downarrow n}=i \frac{\Omega_{n-\beta, n}}{2}\left[\rho_{\downarrow n, \uparrow(n-\beta)}-\rho_{\uparrow(n-\beta), \downarrow n}\right] \\
&+(1-\xi) \Gamma_{\mathrm{eff}} \rho_{\uparrow n, \uparrow n}+\xi \Gamma_{\mathrm{eff}} \rho_{\uparrow(n-1), \uparrow(n-1),} \\
& \dot{\rho}_{\downarrow n, \uparrow(n-\beta)}=-\dot{\rho}_{\uparrow(n-\beta), \downarrow n} \\
&=i \frac{\Omega_{n-\beta, n}}{2}\left[\rho_{\downarrow n, \downarrow n}-\rho_{\uparrow(n-\beta), \uparrow(n-\beta)}\right] \\
&-\frac{\Gamma_{\mathrm{eff}}}{2} \rho_{\downarrow n, \uparrow(n-\beta)}, \\
& \dot{\rho}_{\uparrow(n-\beta), \uparrow(n-\beta)}= i \frac{\Omega_{n-\beta, n}}{2}\left[\rho_{\uparrow(n-\beta), \downarrow n}-\rho_{g n, \uparrow(n-\beta)}\right] \\
&-\Gamma_{\mathrm{eff}} \rho_{\uparrow(n-\beta), \uparrow(n-\beta)},
\end{aligned}
$$

with the density matrix elements defined in the usual way, e.g.,

$$
\rho_{\downarrow n, \uparrow(n-\beta)}=\langle\downarrow, n|\rho| \uparrow,(n-\beta)\rangle
$$

and $\Omega_{n, n-\beta}$ as the effective Rabi frequency coupling the two states $|\downarrow, n\rangle$ and $|\uparrow, n-\beta\rangle$. This system of differential equations is solved numerically to produce the evolution during a single Raman cooling pulse.

For every set of parameters $\left(T_{\mathrm{c}}, \alpha, t_{\mathrm{R} 1}\right.$, and $\left.t_{\mathrm{R} 2}\right)$, the evolution during the pulse sequence as shown in Fig. 3 is computed by repeatedly solving the equations above. The actual evolution time for the atomic system during each pulse is reduced by $1 \mu \mathrm{s}$ from the chosen pulse lengths to include realistic experimental pulse area reduction. The final population in the motional ground state is considered as the signal detected by the STIRAP pulse and corrected by an amplitude $a=0.7$ and an offset $b=0.23$ reduction due to experimental imperfections according to

$$
y=a\left(y_{\text {theory }}-b\right) .
$$

A scan over the SBC cooling time $T_{\mathrm{c}}$ reproduces the experimental data, which are processed in a similar way as described in Sec. II B.
[1] D. Leibfried, B. DeMarco, V. Meyer, D. Lucas, M. Barrett, J. Britton, W. M. Itano, B. Jelenković, C. Langer, T. Rosenband, and D. J. Wineland, Nature (London) 422, 412 (2003).

[2] H. Häffner, C. F. Roos, and R. Blatt, Phys. Rep. 469, 155 (2008).

[3] R. Blatt and D. Wineland, Nature (London) 453, 1008 (2008).

[4] D. J. Wineland, Rev. Mod. Phys. 85, 1103 (2013).

[5] R. Blatt and C. F. Roos, Nat. Phys. 8, 277 (2012).

[6] T. Schaetz, C. R. Monroe, and T. Esslinger, New J. Phys. 15, 085009 (2013).

[7] P. O. Schmidt, T. Rosenband, C. Langer, W. M. Itano, J. C. Bergquist, and D. J. Wineland, Science 309, 749 (2005).

[8] C. F. Roos, M. Chwalla, K. Kim, M. Riebe, and R. Blatt, Nature (London) 443, 316 (2006).

[9] T. Rosenband, D. B. Hume, P. O. Schmidt, C. W. Chou, A. Brusch, L. Lorini, W. H. Oskay, R. E. Drullinger, T. M. Fortier, J. E. Stalnaker, S. A. Diddams, W. C. Swann, N. R.
Newbury, W. M. Itano, D. J. Wineland, and J. C. Bergquist, Science 319, 1808 (2008).

[10] C. Hempel, B. P. Lanyon, P. Jurcevic, R. Gerritsma, R. Blatt, and C. F. Roos, Nat. Photon 7, 630 (2013).

[11] Y. Wan, F. Gebert, J. B. Wübbena, N. Scharnhorst, S. Amairi, I. D. Leroux, B. Hemmerling, N. Lörch, K. Hammerer, and P. O. Schmidt, Nat. Commun. 5, 3096 (2014).

[12] A. D. Ludlow, M. M. Boyd, J. Ye, E. Peik, and P. O. Schmidt, Rev. Mod. Phys. (to be published), arXiv:1407.3493.

[13] J. Mur-Petit, J. Pérez-Ríos, J. Campos-Martínez, M. I. Hernández, S. Willitsch, and J. J. García-Ripoll, in Architecture and Design of Molecule Logic Gates and Atom Circuits, Advances in Atom and Single Molecule Machines, edited by N. Lorente and C. Joachim (Springer, Berlin, 2013), pp. 267-277.

[14] M. Shi, P. F. Herskind, M. Drewsen, and I. L. Chuang, New J. Phys. 15, 113019 (2013). 
[15] S. Willitsch, M. T. Bell, A. D. Gingell, and T. P. Softley, Phys. Chem. Chem. Phys. 10, 7200 (2008).

[16] S. Willitsch, M. T. Bell, A. D. Gingell, S. R. Procter, and T. P. Softley, Phys. Rev. Lett. 100, 043203 (2008).

[17] P. F. Staanum, K. Højbjerre, R. Wester, and M. Drewsen, Phys. Rev. Lett. 100, 243003 (2008).

[18] S. Schiller and V. Korobov, Phys. Rev. A 71, 032505 (2005).

[19] V. V. Flambaum and M. G. Kozlov, Phys. Rev. Lett. 99, 150801 (2007).

[20] K. Beloy, M. G. Kozlov, A. Borschevsky, A. W. Hauser, V. V. Flambaum, and P. Schwerdtfeger, Phys. Rev. A 83, 062514 (2011).

[21] M. Kajita, M. Abe, M. Hada, and Y. Moriwaki, J. Phys. B: At. Mol. Opt. Phys. 44, 025402 (2011).

[22] M. Kajita, G. Gopakumar, M. Abe, M. Hada, and M. Keller, Phys. Rev. A 89, 032509 (2014).

[23] B. Ravaine, S. G. Porsev, and A. Derevianko, Phys. Rev. Lett. 94, 013001 (2005).

[24] E. R. Meyer, J. L. Bohn, and M. P. Deskevich, Phys. Rev. A 73, 062108 (2006).

[25] A. N. Petrov, N. S. Mosyagin, T. A. Isaev, and A. V. Titov, Phys. Rev. A 76, 030501 (2007).

[26] A. Leanhardt, J. Bohn, H. Loh, P. Maletinsky, E. Meyer, L. Sinclair, R. Stutz, and E. Cornell, J. Mol. Spectrosc. 270, 1 (2011).

[27] H. Loh, K. C. Cossel, M. C. Grau, K.-K. Ni, E. R. Meyer, J. L. Bohn, J. Ye, and E. A. Cornell, Science 342, 1220 (2013).

[28] R. Berger, in Theoretical and Computational Chemistry, Relativistic Electronic Structure Theory Part 2. Applications, Vol. 14, edited by P. Schwerdtfeger (Elsevier, New York, 2004), pp. 188288.

[29] M. Quack, J. Stohner, and M. Willeke, Annu. Rev. Phys. Chem. 59, 741 (2008).

[30] R. Bast, A. Koers, A. S. P. Gomes, M. Iliaš, L. Visscher, P. Schwerdtfeger, and T. Saue, Phys. Chem. Chem. Phys. 13, 864 (2010).

[31] B. Darquié, C. Stoeffler, A. Shelkovnikov, C. Daussy, A. AmyKlein, C. Chardonnet, S. Zrig, L. Guy, J. Crassous, P. Soulard, P. Asselin, T. R. Huet, P. Schwerdtfeger, R. Bast, and T. Saue, Chirality 22, 870 (2010).

[32] J. H. V. Nguyen, C. R. Viteri, E. G. Hohenstein, C. D. Sherrill, K. R. Brown, and B. Odom, New J. Phys. 13, 063023 (2011).

[33] J. H. V. Nguyen and B. Odom, Phys. Rev. A 83, 053404 (2011).

[34] C.-Y. Lien, C. M. Seck, Y.-W. Lin, J. H. V. Nguyen, D. A. Tabor, and B. C. Odom, Nat. Commun. 5, 4783 (2014).

[35] T. Baba and I. Waki, Jpn. J. Appl. Phys. 35, L1134 (1996).

[36] M. Welling, H. A. Schuessler, R. I. Thompson, and H. Walther, Int. J. Mass Spectrom. Ion Processes 172, 95 (1998).

[37] B. Roth, A. Ostendorf, H. Wenz, and S. Schiller, J. Phys. B: At. Mol. Opt. Phys. 38, 3673 (2005).

[38] X. Tong, A. H. Winney, and S. Willitsch, Phys. Rev. Lett. 105, 143001 (2010).

[39] J. E. Goeders, C. R. Clark, G. Vittorini, K. Wright, C. R. Viteri, and K. R. Brown, J. Phys. Chem. A 117, 9725 (2013).

[40] R. Rugango, J. E. Goeders, T. H. Dixon, J. M. Gray, N. Khanyile, G. Shu, R. J. Clark, and K. R. Brown, arXiv:1412.0782.

[41] H. Rohde, S. T. Gulde, C. F. Roos, P. A. Barton, D. Leibfried, J. Eschner, F. Schmidt-Kaler, and R. Blatt, J. Opt. B: Quantum Semiclass. Opt. 3, S34 (2001).
[42] M. D. Barrett, B. DeMarco, T. Schaetz, V. Meyer, D. Leibfried, J. Britton, J. Chiaverini, W. M. Itano, B. Jelenković, J. D. Jost, C. Langer, T. Rosenband, and D. J. Wineland, Phys. Rev. A 68, 042302 (2003).

[43] C. W. Chou, D. B. Hume, J. C. J. Koelemeij, D. J. Wineland, and T. Rosenband, Phys. Rev. Lett. 104, 070802 (2010).

[44] J. P. Home, M. J. McDonnell, D. J. Szwer, B. C. Keitch, D. M. Lucas, D. N. Stacey, and A. M. Steane, Phys. Rev. A 79, 050305 (2009).

[45] Y. Lin, J. P. Gaebler, T. R. Tan, R. Bowler, J. D. Jost, D. Leibfried, and D. J. Wineland, Phys. Rev. Lett. 110, 153002 (2013).

[46] C. Roos, T. Zeiger, H. Rohde, H. C. Nägerl, J. Eschner, D. Leibfried, F. Schmidt-Kaler, and R. Blatt, Phys. Rev. Lett. 83, 4713 (1999).

[47] D. Kielpinski, C. Monroe, and D. J. Wineland, Nature (London) 417, 709 (2002).

[48] F. Diedrich, J. C. Bergquist, W. M. Itano, and D. J. Wineland, Phys. Rev. Lett. 62, 403 (1989).

[49] C. Monroe, D. M. Meekhof, B. E. King, S. R. Jefferts, W. M. Itano, D. J. Wineland, and P. Gould, Phys. Rev. Lett. 75, 4011 (1995).

[50] C. Roos, Controlling the quantum state of trapped ions, Ph.D. thesis, University of Innsbruck, 2000.

[51] J. Eschner, G. Morigi, F. Schmidt-Kaler, and R. Blatt, J. Opt. Soc. Am. B 20, 1003 (2003).

[52] S. E. Hamann, D. L. Haycock, G. Klose, P. H. Pax, I. H. Deutsch, and P. S. Jessen, Phys. Rev. Lett. 80, 4149 (1998).

[53] V. Vuletić, C. Chin, A. J. Kerman, and S. Chu, Phys. Rev. Lett. 81, 5768 (1998).

[54] J. D. Thompson, T. G. Tiecke, A. S. Zibrov, V. Vuletić, and M. D. Lukin, Phys. Rev. Lett. 110, 133001 (2013).

[55] A. Schliesser, R. Rivière, G. Anetsberger, O. Arcizet, and T. J. Kippenberg, Nat. Phys. 4, 415 (2008).

[56] J. D. Teufel, T. Donner, D. Li, J. W. Harlow, M. S. Allman, K. Cicak, A. J. Sirois, J. D. Whittaker, K. W. Lehnert, and R. W. Simmonds, Nature (London) 475, 359 (2011).

[57] J. Chan, T. P. M. Alegre, A. H. Safavi-Naeini, J. T. Hill, A. Krause, S. Gröblacher, M. Aspelmeyer, and O. Painter, Nature (London) 478, 89 (2011).

[58] D. J. Wineland and W. M. Itano, Phys. Rev. A 20, 1521 (1979).

[59] J. Javanainen and S. Stenholm, Appl. Phys. A 24, 151 (1981).

[60] S. Stenholm, Rev. Mod. Phys. 58, 699 (1986).

[61] I. Marzoli, J. I. Cirac, R. Blatt, and P. Zoller, Phys. Rev. A 49, 2771 (1994).

[62] D. J. Heinzen and D. J. Wineland, Phys. Rev. A 42, 2977 (1990).

[63] D. J. Wineland, C. Monroe, W. M. Itano, D. Leibfried, B. E. King, and D. M. Meekhof, J. Res. Natl. Inst. Stand. Technol. 103, 259 (1998).

[64] G. Morigi, J. I. Cirac, M. Lewenstein, and P. Zoller, EPL 39, 13 (1997).

[65] D. Stevens, J. Brochard, and A. M. Steane, Phys. Rev. A 58, 2750 (1998).

[66] G. Morigi, J. Eschner, J. I. Cirac, and P. Zoller, Phys. Rev. A 59, 3797 (1999).

[67] B. Hemmerling, F. Gebert, Y. Wan, D. Nigg, I. V. Sherstov, and P. O. Schmidt, Appl. Phys. B 104, 583 (2011).

[68] F. Gebert, Precision measurement of the isotopic shift in calcium ions using photon recoil spectroscopy, Ph.D. thesis, Leibniz Universität Hannover, 2015. 
[69] Probing the population using a BSB instead of a RSB STIRAP pulse allows for detection of population loss into other hyperfine states by reducing the signal.

[70] S. Stenholm, J. Opt. Soc. Am. B 2, 1743 (1985).

[71] Q. A. Turchette, D. Kielpinski, B. E. King, D. Leibfried, D. M. Meekhof, C. J. Myatt, M. A. Rowe, C. A. Sackett, C. S. Wood, W. M. Itano, C. Monroe, and D. J. Wineland, Phys. Rev. A 61, 063418 (2000).

[72] N. Kjaergaard, L. Hornekaer, A. M. Thommesen, Z. Videsen, and M. Drewsen, Appl. Phys. B 71, 207 (2000).

[73] K. Mølhave and M. Drewsen, Phys. Rev. A 62, 011401 (2000).

[74] M. Drewsen, A. Mortensen, R. Martinussen, P. Staanum, and J. L. Sørensen, Phys. Rev. Lett. 93, 243201 (2004).

[75] J. B. Wübbena, S. Amairi, O. Mandel, and P. O. Schmidt, Phys. Rev. A 85, 043412 (2012).
[76] P. O. Schmidt, T. Rosenband, J. C. J. Koelemeij, D. B. Hume, W. M. Itano, J. C. Bergquist, and D. J. Wineland, AIP Conf. Proc. 862, 305 (2006).

[77] I. S. Vogelius, L. B. Madsen, and M. Drewsen, J. Phys. B: At. Mol. Opt. Phys. 39, S1259 (2006).

[78] J. C. J. Koelemeij, B. Roth, and S. Schiller, Phys. Rev. A 76, 023413 (2007).

[79] C. L. Arrington, K. S. McKay, E. D. Baca, J. J. Coleman, Y. Colombe, P. Finnegan, D. A. Hite, A. E. Hollowell, R. Jördens, J. D. Jost, D. Leibfried, A. M. Rowen, U. Warring, M. Weides, A. C. Wilson, D. J. Wineland, and D. P. Pappas, Rev. Sci. Instrum. 84, 085001 (2013).

[80] The spontaneous decay during the Raman cooling cycle is either caused by the off-resonant excitation from the Raman lasers or via the rf coupling and the ${ }^{2} P_{1 / 2}$ laser. 